\title{
Polyphenol characterization, anti-oxidant, anti-proliferation and anti-tyrosinase activity of cranberry pomace
}

\author{
Indu Parmar, Sandhya V. Neir, and H.P. Vasantha Rupasinghe*
}

Department of Plant, Food, and Environmental Sciences, Faculty of Agriculture, Dalhousie University, Truro, Nova Scotia, B2N 5E3, Canada

*Corresponding author: Department of Plant, Food, and Environmental Sciences, Faculty of Agriculture, Dalhousie University, Truro, Nova Scotia, B2N 5E3, Canada

Submission Date: September 5, 2016, Accepted Date: November 27, 2016, Publication Date: November 30, 2016

Citation: Parmar I., Neir S.V., and Rupasinghe HPV. Polyphenol characterization, anti-oxidant, anti-proliferation and anti-tyrosinase activity of cranberry pomace. Functional Foods in Health and Disease 2016; 6(11):754-768

\begin{abstract}
Background: Cranberry pomace (CP), an underutilized by-product from juice processing, contains a wide range of biologically active compounds that can be recovered and used in a variety of applications in functional foods and nutraceuticals.
\end{abstract}

Methods: In this study, analytical chemical techniques such as solvent extractions and characterization of extracts in respect with their phenolic content were performed using ultrahigh performance liquid chromatography mass spectrometry (UPLC-MS) and spectrophotometry. Crude CP extract and its phenolic acids, flavonols, anthocyanins and proanthocyanidins-rich fractions were then evaluated for their anti-oxidant capacity, tyrosinase inhibitory activity, and anti-proliferation activity against hepatocellular carcinoma HepG2 cells.

Results: On a dry weight basis, the different $\mathrm{CP}$ fractions contained seven major anthocyanins $(0.1-125 \mathrm{mg} / \mathrm{g})$, six major phenolic acids $(0.8-31 \mathrm{mg} / \mathrm{g})$, seven flavonols $(1-126 \mathrm{mg} / \mathrm{g})$ and five flavan-3-ols $(0.1-12 \mathrm{mg} / \mathrm{g})$. Fractions rich in flavonols exhibited the most potent antioxidant capacities with ferric ion reducing antioxidant power values of 1.8-1.9 mmole/g and 2, 2diphenyl-1-picrylhydrazyl radical scavenging $\mathrm{IC}_{50}$ values of $15.1-15.2 \mathrm{mg} / \mathrm{L}$ respectively. On the other hand, fractions rich in phenolic acids and flavan-3-ol monomers demonstrated the most potent anti-tyrosinase activity $\left(\mathrm{IC}_{50}=6.1-6.2 \mathrm{mg} / \mathrm{L}\right)$ and anti-proliferative activity $\left(\mathrm{IC}_{50}=7.8-15.8\right.$ $\mathrm{mg} / \mathrm{L})$. Generally, all the fractions exhibited a dose-response relationship in the selected biological activity assays. 
Conclusion: This study suggests an effective utilization of CP to obtain biologically active fractions with potential to be used in functional foods and nutraceuticals designed for the prevention of chronic diseases associated with oxidative stress.

Keywords: cranberry pomace, polyphenol, anti-tyrosinase, anti-proliferation, nutraceuticals, functional foods

\section{BACKGROUND}

Cranberry (Vaccinium macrocarpon) is native to North America, although Canada is the second largest producer of cranberries next to the United States of America, with a production of 174,252 tons in 2014 according to Statistics Canada [1]. Cranberry holds a great importance in the Canadian fruit market through its use in manufacture of juice, sauces, jams, marmalades, minced meat, or sweetened dried cranberries. Juice production results in the removal of fibrous skin, seeds and stems from cranberries, which are collectively called cranberry pomace (CP). $\mathrm{CP}$ is generally discarded into landfills due to its low nutritional value such as protein content, which hampers its use in animal feed. Due to its low $\mathrm{pH}$ and high organic matter, its landfill disposal presents a serious environmental challenge, thereby, urging to explore alternative uses of CP. However, CP contains an assortment of beneficial phytochemicals, such as polyphenols, which have a wide potential in functional foods and nutraceutical applications. Previous studies have tried to elucidate the polyphenol characterization of CP. For example, a study by $\mathrm{Vu}$ and colleagues [2] demonstrated that CP contains water soluble anthocyanins and non-polar phenolic compounds such as proanthocyanidins. Roopchand and colleagues (3) attempted to develop a food compatible method for polyphenol extraction and stabilization. Additionally, a few other studies on polyphenolic composition and antioxidant capacity of $\mathrm{CP}$ have reported several phenolic classes $[4,5]$. However, these studies either did not characterize the polyphenolics comprehensively by fractionating each sub-class or did not evaluate the biological properties of the polyphenolic extracts.

The potential use of such polyphenols for human health promotion is widely reported, as these dietary bioactives may play a role in regulating cellular processes related to apoptosis, inflammation, and proliferation, and thereby may be able to prevent oxidative damage and protect against some cancers [6]. Reports on anti-carcinogenic properties of cranberry fruit proanthocyanidins have been emerging in the literature [7, 8]. Additionally, cranberry press-cake has been demonstrated to delay the growth and inhibit metastasis of human breast tumor MDAMB-435 cells [9]. Furthermore, mild anti-proliferative action of different polyphenol fractions from $\mathrm{CP}$ has been suggested against two colon cancer cell lines, HT-29, and LS-513 [2]. Nevertheless, the anti-carcinogenic properties of $\mathrm{CP}$ extracts, the profile of the phenolic compounds, and underlying mechanisms that provide protection against tumors are not as well understood. In light of this, this study attempted to expand further applications of specific polyphenol fractions from $\mathrm{CP}$ with their potential as anti-carcinogenic agents against hepatocellular carcinoma in vitro, cosmetic agents through anti-tyrosinase activity and natural anti-oxidants. 


\section{METHODS}

\section{Raw material}

$\mathrm{CP}$ of Vaccinium macrocarpon was obtained from Cranberry Acres, Berwick, NS. The pomace comprised of the remaining wet hulls after the juice was squeezed out. The pomace, obtained as three independent batches of approximately $30 \mathrm{~kg}$ each was considered as three replicates and was kept at $-20^{\circ} \mathrm{C}$ until use.

\section{Chemicals and reagents}

The liquid chromatography standards used for the study were obtained as follows: quercetin-3$O$-rhamnoside and quercetin-3-O-galactoside were from Indofine Chemical Co. (Hillsborough, N.J., U.S.A.); quercetin-3-O-glucoside, phloridzin, caffeic acid, ferulic acid, protocatechuic acid, myrecetin and chlorogenic acid were from Sigma-Aldrich; quercetin-3- $O$-rutinoside, (-)epicatechin, (+)-catechin, and procyanidin B1 and B2 were from ChromaDex (Santa Ana, CA, U.S.A.); and cyanidin-3-O-galactoside was obtained from Extra-Synthase (Paris, France). Ethanol anhydrous was obtained from Commercial Alcohols (Montreal, QC, Canada). Human hepatocellular carcinoma (HepG2) cell line was purchased from American Type Culture Collection (Cederlane, Burlington, ON, Canada). Unless and otherwise stated, all other chemicals, reagents and enzymes used in this study were procured from Sigma Aldrich (Oakville, ON, Canada).

\section{Cell culture}

Human hepatocellular carcinoma cells HepG2 (ATCC ${ }^{\circledR} \mathrm{HB}-8065^{\mathrm{TM}}$ ), classified as biosafety level I, were obtained from Cedarlane Labs (Burlington, ON, Canada). The cells were maintained in Eagle's Minimum Essential Medium (EMEM) complete growth medium, supplemented with $10 \%$ fetal bovine serum, 100 units $/ \mathrm{mL}$ penicillin and $100 \mu \mathrm{g} / \mathrm{mL}$ streptomycin in a humidified incubator $\left(5 \% \mathrm{CO}_{2}\right)$ at $37^{\circ} \mathrm{C}(\mathrm{VWR}$ International, Mississauga, ON, Canada).

\section{Extraction and isolation of polyphenol fractions}

$\mathrm{CP}$ (500 g FW) was homogenized with equal volume of deionized water (DI) using a blender (Bead Beater; BioSpec Products, Inc. Bartlesville, OK, USA). The fat components were removed from the slurry by $1 \mathrm{~L}$ hexane extraction overnight, followed by liquid-liquid separation. The hexane fraction was discarded and the aqueous mixture was further used for phenolic extraction. The phenolic extraction was carried out using 2 L of acetone/water/acetic acid mix (70/29/1) overnight with continuous stirring at room temperature. The mixture was vacuum filtered and the residue was extracted again using the above explained procedure. After vacuum filtration, both the supernatants were mixed and subjected to rotary vacuum evaporation (Rotavapor, R-200, Buchi, Flawil, Switzerland) at $37^{\circ} \mathrm{C}$ until completely dried. The dried extract was dissolved in $200 \mathrm{~mL}$ of $50 \%$ ethanol in DI water and loaded on to Sepabead column, which was maintained at $25 \%$ ethanol and loaded on a $400 \mathrm{~g}$ adsorbent resin (Sorbent SP-207-05 Sepabead resin brominated, $250 \mu \mathrm{m}$, Sorbent Tech., Norcross, GA, USA) packed into a chromatography column $(46 \times 2.3 \mathrm{~cm}$, length and internal diameter). The column was equilibrated with deionized water and maintained in $25 \%$ ethanol in water. The column was washed with four bed volumes of 
water $(1 \mathrm{~L}$ each) to remove all water-soluble compounds, including sugars, until the Brix value of washed eluent reached $<0.01$. The water wash eluent was collected for further analysis. The phenolic compounds were eluted with $25,50,60,70,80$ and 100\% ethanol (1 L each) to yield six fractions E1-E6 respectively. This was followed by the addition of 25, 50, 60, 70, 80, 90 and $100 \%$ acetone to give additional seven fractions A1-A7 respectively. The column was protected from light during the entire process. All the fractions were stored at $-20^{\circ} \mathrm{C}$ until dried completely using rotary vacuum evaporator.

\section{Spectrophotometric measurements}

\section{Total phenolic content}

The Folin-Ciocalteu assay was used to measure the total phenolic content as described by Singleton and Rossi (10) and modified by Rupasinghe et al. [11]. Calibration curves were plotted using standards at concentrations of $10-750 \mu \mathrm{mol} / \mathrm{L}$ and the results were expressed as gallic acid equivalents (GAE)/g dried weight (DW) of each fraction.

\section{Total anthocyanins}

Total anthocyanins were determined using the $\mathrm{pH}$ differential method described by Lee et al. [12]. The absorbance was measured at 510 and $700 \mathrm{~nm}$ using two buffer systems: potassium chloride buffer $\mathrm{pH} 1.0(0.025 \mathrm{M})$ and sodium acetate buffer $\mathrm{pH} 4.5(0.4 \mathrm{M})$. Total anthocyanins were calculated as equivalents of malvidin-3-glucoside (M3G) according to the equation [1].

Total anthocyanin $(\mathrm{mg} / \mathrm{L})=(\mathrm{A} \times \mathrm{MW} \times \mathrm{DF} \times 1000) /(\varepsilon \times 1.0)$

where $\mathrm{A}=(\mathrm{A} 550-\mathrm{A} 700$, at $\mathrm{pH} 1)-(\mathrm{A} 550-\mathrm{A} 700$, at $\mathrm{pH} 4.5) ; \mathrm{MW}=$ molecular weight of $\mathrm{M} 3 \mathrm{G}=493.2 \mathrm{~g} /$ mole; $\mathrm{DF}$ is dilution factor ; $\varepsilon=$ Molar extinction coefficient 28,$000 ; 1$ is path length $(1 \mathrm{~cm})$ and 1000 is factor for conversion from $\mathrm{g}$ to $\mathrm{mg}$.

\section{Total proanthocyanidin content}

For total proanthocyanidins content, dimethylcinnamaldehyde (DMAC) method was used as described by Prior et al. [13] and modified by Bhullar and Rupasinghe [14]. Calibration curves of standard were plotted using catechin at $10-500 \mathrm{mg} / \mathrm{L}$.

\section{Total flavonoid content}

The total flavonoid content was determined using aluminum chloride colorimetric method [15]. The results were expressed as $\mu$ mole quercetin equivalents $(\mathrm{QE}) / 100 \mathrm{~g}$ FW using standard calibration curve at concentrations of $10-500 \mu \mathrm{mol} / \mathrm{L}$.

\section{UPLC-ESI-MS analysis}

Out of each dried fraction, $10 \mathrm{mg}$ was weighed out and dissolved in $10 \mathrm{~mL}$ methanol-acetone [7:3]. The extract was further diluted 10 times prior to filtration through $0.22 \mu$ m nylon filters and placement into auto-sampler vials. An ultra-high performance liquid chromatography (UPLC) (Model H-class system, Waters, Milford, MA, USA) equipped with an acuity UPLC BEH C $_{18}$ column $(2.1 \times 100 \mathrm{~mm}, 1.7 \mu \mathrm{m})$ (Waters, Milford, MA, USA) was used for analysis. MS analysis was performed with a Micromass Quattro micro API MS/MS system, which is 
controlled by MassLynx V4.1 data analysis system (Micromass, Cary, NC, USA). The injection volume of each sample was maintained at $2 \mu \mathrm{L}$. All the standards and samples were prepared in $100 \%$ methanol with concentration range of $0.20-20 \mathrm{mg} / \mathrm{L}$. The anthocyanin standard samples were also prepared in methanol with a concentration range between $0.25-25 \mathrm{mg} / \mathrm{L}$ for all the standards used. MS-MS analysis was carried out using a Micro-mass Quattro Micro API MS/MS system. Electro spray ionization (ESI), in negative ion mode (ESI-), was used for the analysis of the flavonols, flavan-3-ol, and phenolic acid compounds. ESI in positive ion mode was used for anthocyanins. Mass spectrometry conditions used were as follows: capillary voltage $3000 \mathrm{~V}$, nebulizing gas $\left(\mathrm{N}_{2}\right)$ at a temperature of $375{ }^{\circ} \mathrm{C}$ at a flow rate of $0.35 \mathrm{~mL} \mathrm{~min}^{-1}$. The cone voltage $(25-50 \mathrm{~V})$ was optimized for each compound. Individual compounds were identified using the multiple reactions monitoring mode (MRM), using specific precursor-production transitions as reported previously [14].

\section{Total antioxidant capacity}

a) The ferric reducing antioxidant power (FRAP) assay

The FRAP assay was used to determine the electron donating potential of the CP samples based on the assay described by Benzie and Strain [16] and modified by Rupasinghe et al. [15] and the results were expressed as $\mu$ mol Trolox equivalents (TE)/100 g DM using a standard curve of Trolox with a concentration range of $10-500 \mu \mathrm{mol} / \mathrm{L}$.

\section{b) 2,2-Diphenyl-1-picrylhydrazyl (DPPH) radical scavenging assay}

The free radical scavenging activity of CP polyphenols was measured using DPPH method described by $\mathrm{Lu}$ and Foo (17) and modified by Rupasinghe et al. (15) The $\mathrm{IC}_{50}$ value, defined as the amount of the sample to scavenge 50\% of the DPPH radicals, was calculated from the results.

\section{HepG2 cell viability assay}

All the fractions were dried using a rotary vacuum evaporator until a completely dried powder was obtained. All acetone fractions were pooled together and were given label 'A'. From each extract, $10 \mathrm{mg}$ of the sample was dissolved using dimethyl sulfoxide (DMSO), and five different dilutions were prepared in eagle's minimal essential media (EMEM) for cell study. The extracts from all fractions were used to measure their ability to inhibit human liver cancer cell proliferation using HepG2 cell lines [18]. The cell cultures were exposed to various concentrations of the extracts during a 24-h growth period. The anti-proliferative activity of the CP extracts was measured by the ability of viable cells to reduce 3-(4, 5-dimethylthiazol-2-yl)-5(3-carboxymethoxyphenyl)-2-(4-sulfenyl)-2Htetrazolium (MTS) to formazan. This product absorbs light at $490 \mathrm{~nm}$ and the absorbance was measured using a BMG Fluostar microplate reader (Mandel, QC, Canada). Six replications for each treatment were used to determine the inhibitory effect on cell proliferation. The effective median dose $\left(\mathrm{EC}_{50}\right)$ was calculated and expressed as $\mathrm{mg} / \mathrm{mL}$.

\section{Anti-tyrosinase activity}

The anti-tyrosinase effect of each fraction was determined based on the inhibition of the conversion of specific substrate of tyrosinase, L-DOPA into colored product dopachrome, which has maximum absorption at $492 \mathrm{~nm}$. The procedure used was described by Sirat et al. [19] and modified by Ziaullah et al. [20]. The concentration of the fractions demonstrating 50\% activity 
were calculated as an inhibitory concentration $\left(\mathrm{IC}_{50}\right)$ and compared with a control in terms of mean \pm standard deviation (SD).

\section{Statistical analysis}

A completely randomized design (CRD) was used and all experiments were done in triplicates $(n=3)$. All measurements were conducted in triplicates and the means were reported. Analysis of variance (ANOVA) was used to test the significance of each variable $(p<0.05)$ and the multiple mean comparison was performed in General Linear Model (SAS V8, Cary, NC, USA) of SAS using Tukey's test method. The differences at the $5 \%$ level $(\mathrm{p}<0.05)$ were considered statistically significant.

\section{RESULTS AND DISCUSSION}

\section{Spectrophotometric analyses of polyphenols}

The total phenolic content of the crude CP extract as measured by Folin Ciocalteu method was $451 \mu$ mole GAE/g DW (Figure 1).
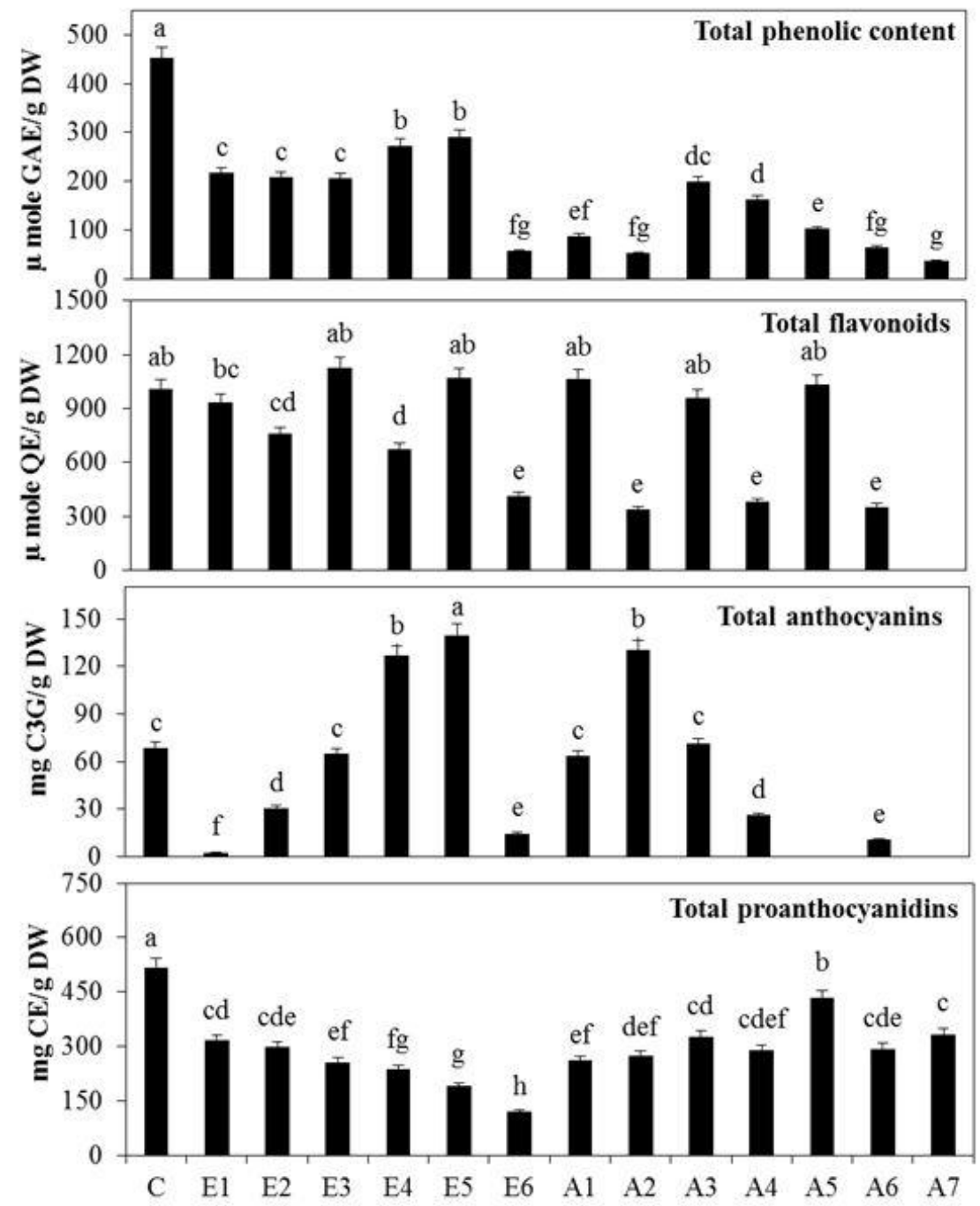

Figure 1. Total phenolic, total flavonoid and total proanthocyanidin content of each fraction from cranberry pomace. C, crude; E1-E6, ethanol based fractions; A1-A7, acetone based fractions. GAE: gallic acid equivalents; QE: quercetin equivalents; C3G: cyanidin-3-glucoside equivalents; $C E$ : catechin equivalents. 
Further fractionation was carried out and divided as following based on analytical assays: E1 and E2 rich in phenolic acids and flavan-3-ol monomers, E3-E5 rich in flavonols and anthocyanins, and A1-A7 rich in oligomeric and polymeric proanthocyanidins. Depending on the solvent to water ratio used for the extraction process, the phenolic content varied from 37 to 290 $\mu$ mole GAE/g of extract. The total phenolic content for ethanol and acetone fractions was found to lie within ranges from 57-290 $\mu$ mole GAE/g DW and 36-200 $\mu$ mole GAE/g DW respectively. Flavonols and anthocyanin-rich fractions, E4-E5 contained polyphenol content of 272-290 $\mu$ mole GAE/g DW, which was significantly higher than all other fractions. Fractions E4 and E5 added together contained $560 \mu$ mole GAE/g DW, thereby representing about $24 \%$ higher amount than the crude extract.

Total anthocyanins as measured by $\mathrm{pH}$ differential method were significantly higher in fractions E3-E5 than all the other fractions, representing more than 50\% of the total anthocyanins obtained from all fractions. As expected, E1 and E6 contained the least amount of anthocyanins. However, owing to acid hydrolysis employed in the $\mathrm{pH}$ differential method, acetone based fractions (rich in proanthocyanidins) showed varying anthocyanin content.

For total proanthocyanidins, the acetone based fractions contained higher amounts than the ethanol fractions (Figure 1). The catechin equivalents found in ethanol fractions, were presumably due to monomeric flavan-3-ol and/or dimeric proanthocyanidin elution in solvents such as ethanol. The crude extract had the highest amount of procyanidin content, because of the presence of monomeric, dimeric, and oligomeric proanthocyanidins. A previous study has reported the presence of both A- and B-type linkages in cranberry procyanidins [5].

The total flavonoid content of the fractions varied from 415-1128 $\mu$ mole QE/g DW (Figure 1). The crude extract contained flavonoid content of $1000 \mu$ mole QE/g dried fraction, which was significantly higher than most of the ethanol and acetone based fractions. Similar to anthocyanin data, fractions E3 and E5 were observed as the highest total flavonoid containing fractions, followed by fractions E1, E2, and E4.

\section{UPLC-MS characterization of polyphenols}

The LCMS analysis of polyphenols resulted in varying amounts than spectrophotometric measurements (Figure 2). This could be partly due to dissimilar absorbance of different phenolic compounds in the spectrophotometric procedures, but it also indicates the presence of unidentified phenolic compounds in the extracts, for example, benzoic acids and tannins. Most of the dihydrochalcones (phloridzin, phloretin), phenolic acids (protocatechuic acid, chlorogenic acid, caffeic acid, ferulic acid, isoferulic acid), flavonols (myrecetin, quercetin and its glactosides, glucosides, rhamnosides and rutinosides), anthocyanins (glucosides, galactosides and rutinosides of cyanidin, malvidin, delphinidin and peonidins), monomeric flavan-3-ols (catechin, epicatechin, epigallocatechin, epigallocatechin gallate) were concentrated in fractions from E1-E6.

Similar to the results described above, the crude fraction contained the highest amount of most of these compounds, except for total anthocyanins. However, the total phenolic compounds (sum of total phenolic acids, flavonols, catechins, flavan-3-ols and anthocyanins from UPLCESI-MS analysis) were the highest in fractions E4 and E5, representing values of 274 and 227 
$\mathrm{mg} / \mathrm{g}$ DW respectively. Fractions E4 and E5 combined together represented about 43\%, 27\% and $55 \%$ of the total dihydrochalcones, phenolic acids, and total flavonols respectively.
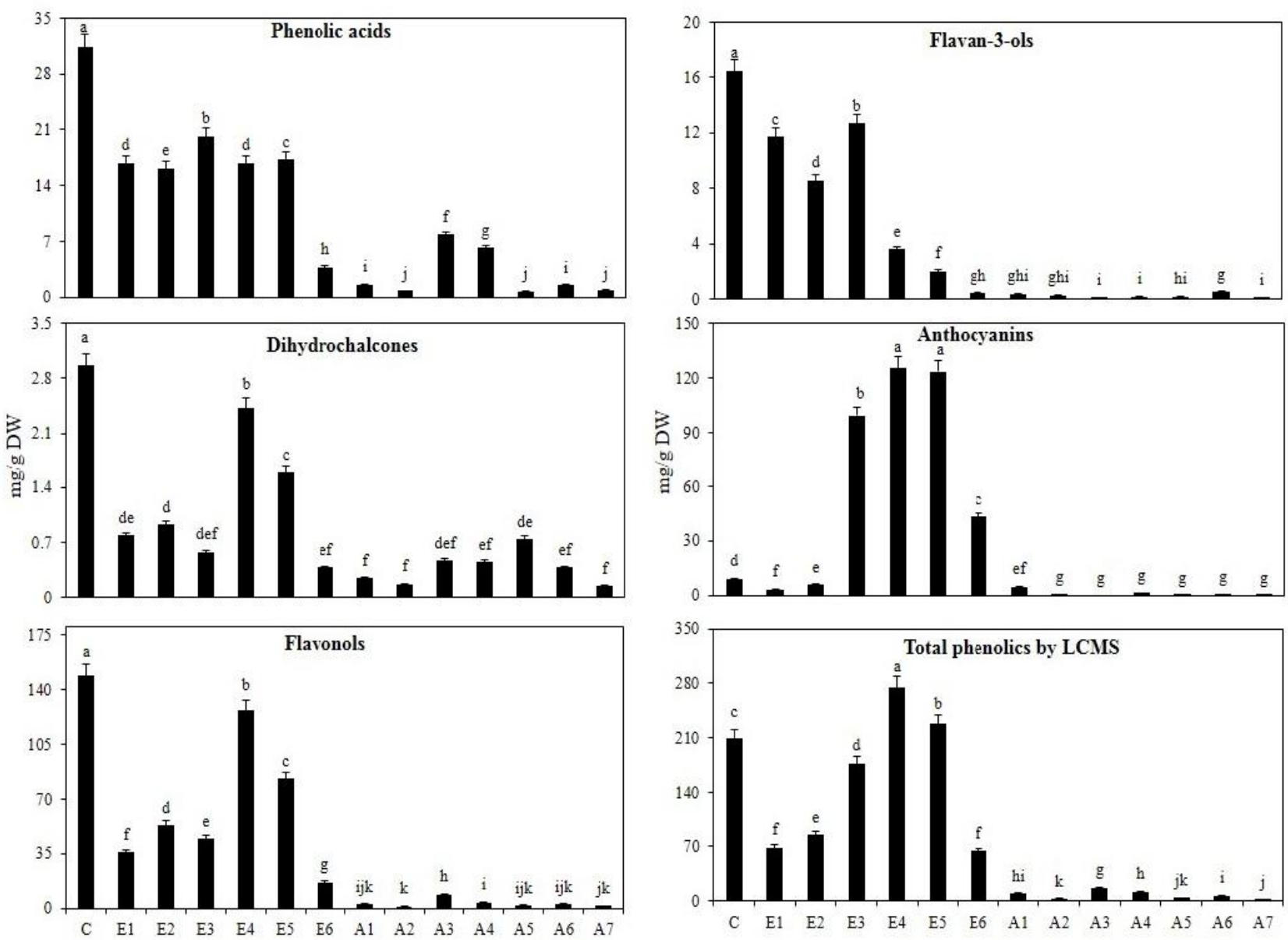

Figure 2. UPLC-ESI-MS analysis of each fraction obtained from cranberry pomace.

Bars represent standard errors $(\mathrm{n}=3)$. Bars with different letters indicate that values are statistically different $(\mathrm{p}$ <0.05). C, crude; E1-E6, ethanol based fractions; A1-A7, acetone based fractions. Dihydrochalcones: phloridzin+phloretin; Phenolic acids: coumaric acid+protocatechuic acid+chlorogenic acid+caffeic acid+ferulic acid+isoferulic acid; total flavonols: myrecetin+quercetin $(\mathrm{Q})+\mathrm{Q}$. galactoside+Q. glucoside+Q. arabinoside+Q. rhamnoside+Q. rutinoside; total flavan-3-ols: catechin+epicatechin+epicatechin gallate+epigallocatechin gallate; Anthocyanins:delphinidin-3-rutinoside+delphinidin-3-galactoside+cyanidin-3-rutinoside+cyanidin-3-galactoside +peonidin-3-galactoside+malvinidin-3-galactoside.

However, the total flavan-3-ol content of fraction E4 and E5 was significantly lower than the other ethanol fractions. Overall, the fractions E4 and E5 represented about $254.4 \mathrm{mg}$ of total phenolics as quantified by UPLC-ESI-MS per g DW. About $83 \%$ of the total phenolics in E4 and E5 as measured by UPLC-ESI-MS were represented by flavonols. Similar to a previous report (White et al., 2010), most of the flavonols found were glycosides of quercetin and myrcetin, although moderate amounts of aglycones were observed as well. Our UPLC-ESI-MS data were consistent with previous reports which showed the presence of the measured different classes of phenolic compounds in by-products of cranberry-juice processing [21, 22].

Characterization by UPLC-ESI-MS provided an insight about multiple anthocyanin glycosides in CP (Figure 2), including the galactosides and glucosides of cyanidin, malvidin, 
peonidin, delphidins and rutinosides of cyanidin, and delphinidin. Interestingly, rutinosides of delphinidin and cyanidins were the most prominent anthocyanins, followed by glucoside and galactoside of delphinidin. Anthocyanins started eluting after fraction E2; therefore, matched with the $\mathrm{pH}$ differential method results, fractions E3-E5 were found rich in most types of anthocyanins. Similar to the previous studies, our report demonstrated the occurrence of galactosides of peonidin and cyanidin; however, no analysis on the arabinosides of peonidin and cyanidins was made as reported in previous studies.

\section{Anti-oxidant capacity}

The FRAP data revealed that almost all the fractions are free radical-scavengers and primary antioxidants, which combat the free radicals (Figure 3).
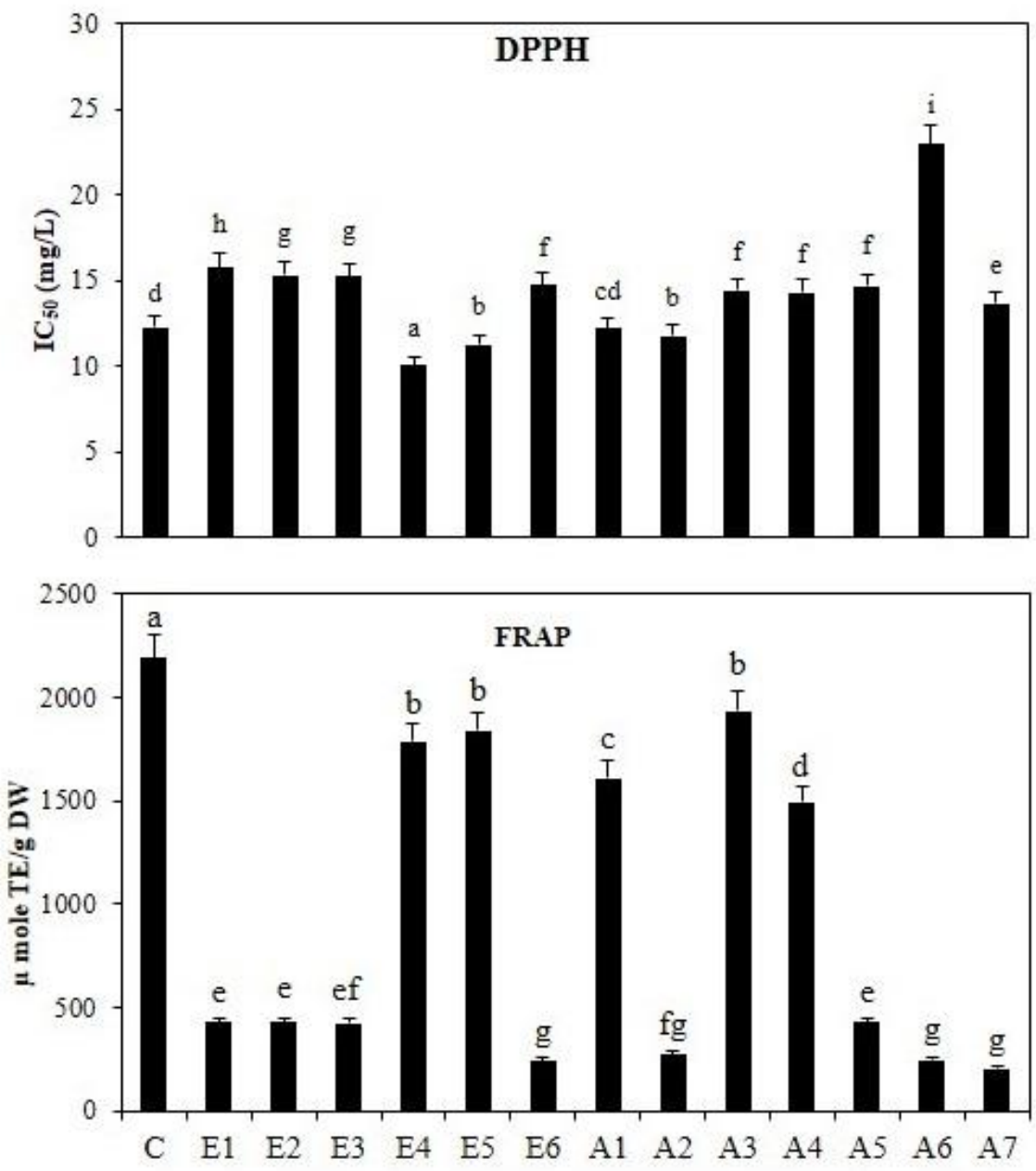

Figure 3. DPPH radical-scavenging capacity and FRAP of each fraction from cranberry pomace. Bars represent standard errors $(\mathrm{n}=3)$. Bars with different letters indicate that values are statistically different $(\mathrm{p}$ $<0.05)$. C, crude; E1-E6, ethanol based fractions; A1-A7, acetone based fractions. $\mathrm{IC}_{50}$ : concentration required to scavenge $50 \%$ of the DPPH radicals; TE: Trolox equivalents

Fraction A1 (rich in proanthocyanidins) contained the highest total FRAP content $(2609.4 \mu$ mol TE/g DW), which was about 40\% higher than E4 and E5 fractions, which were rich in flavonols 
and dihydrochalcones. Additionally, other hydrophobic fractions, A3 and A4 (containing proanthocyanidins), were also found to have high FRAP values. Conversely, fractions rich in phenolic acids (E1-E3) represented the lowest FRAP values of $<500 \mu$ mole TE/g DW. These data results are in agreement with a recent study which showed that large polyphenols (i.e. proanthocyanidins and flavonoid glycosides) containing fractions of cranberry showed the highest anti-oxidant activity [7] among all fractions. It is established that a wide range of phenolics in cranberry known to contribute to the characteristic antioxidant activity profile include the catechins, quercetin, $p$-coumaric acid, chlorogenic acid, myricetin, trans-resveratrol, and cyanidin/peonidin-3-galactoside/arabinoside [23]. Moreover, the capability of a particular phenolic compound towards the total antioxidant capacity of cranberry and its by-products could also depend on both the relative concentration of individual compounds, as well as possible synergistic action of different antioxidant compounds in the fruit [23].

Similar to FRAP, the $\mathrm{IC}_{50}$ values for DPPH assay for the fraction E4 and E5 were the lowest amongst all fractions, representing 10 and $11 \mathrm{mg} / \mathrm{L}$ respectively (Figure 3). The $\mathrm{IC}_{50}$ value for the crude extract was $12.3 \mathrm{mg} / \mathrm{L}$. The correlation coefficient between DPPH radical scavenging activity and reducing power is rather small, indicating some compounds which demonstrated high DPPH scavenging activity may not show reducing power activity due to the fact that ferrous reducing power is a method for measuring total reducing power of electron donating substances, while DPPH assay is a method for measuring the ability of antioxidant molecules to quench DPPH free radical.

\section{Anti-proliferative activity against human hepatocellular carcinoma HepG2 cells}

It is a well-known fact that liver is one of the main sites of flavonoid metabolism. Therefore, it is interesting to see the effects of flavonoid and phenolic acid rich fractions against human hepatocellular carcinoma cell line HepG2. Anti-proliferative activity of fractions E2 was the highest ( $\geq 85 \%$ at a concentration of $100 \mathrm{mg} / \mathrm{L}$ ), followed by fractions $\mathrm{E} 1$, E6, and C, that were nonsignificantly different from each other (Appendix A). Even at lower concentrations of 10 and 25 $\mathrm{mg} / \mathrm{L}$, fractions E1 and E2 demonstrated anti-proliferation of 50\% and above. In general, a doseresponse relationship was observed between the tested fractions and their associated antiproliferative action. The $\mathrm{IC}_{50}$ values $(\mathrm{mg} / \mathrm{L})$ for the tested fractions were as following: $\mathrm{E} 1$ $(7.8)<\mathrm{E} 2(15.8)<\mathrm{E} 3(20.1)<\mathrm{E} 4=\mathrm{C}(62.3)<\mathrm{C}(63.6)<\mathrm{E} 6(77.8)=\mathrm{A}(77.9)<\mathrm{E} 5$ (93.5) as shown in Figure 4. The results suggested that E1 and E2 being rich in short chain phenolic acids and monomeric flavan-3-ols, were able to penetrate through the cell membrane efficiently, thereby giving higher anti-proliferation activity. The present data support a study by $\mathrm{Vu}$ et al. [2], which demonstrated that water soluble phenolic extracts of cranberry and its products (mainly phenolic acids and their derivatives) effectively inhibited the proliferation of HT-29 and LS-513 colon cancer cells. Furthermore, these phenolic acids such as dicaffeoylquinic acid have been shown to exhibit anti-tumor activity against liver cancer [24]. It can be suggested that the observed antiproliferative activity of given $\mathrm{CP}$ extracts could be due to the combined effects of several bioactive compounds present in the fractions, and this symbiotic action can be better than any single compound. Therefore, the results obtained in our study on the anti-proliferative activity of extracts containing polar phenolic acids and flavan-3-ol monomers against HepG2 liver cancer 
cells are scientifically reasonable. However, further investigations are required to confirm our observations.

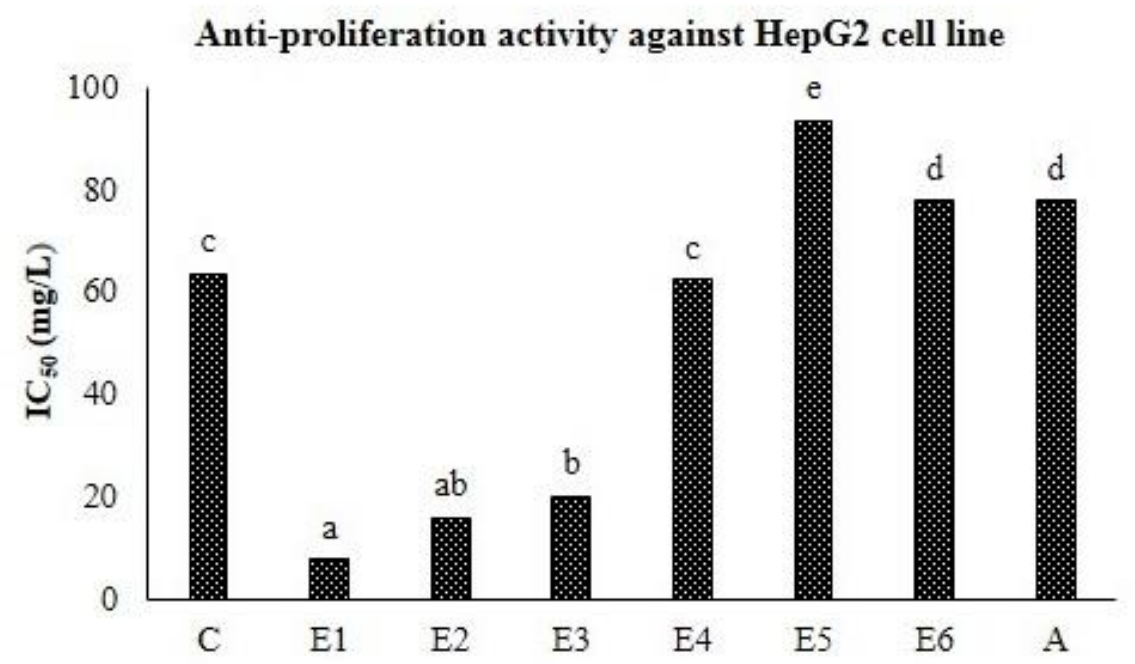

Anti-tyrosinase activity

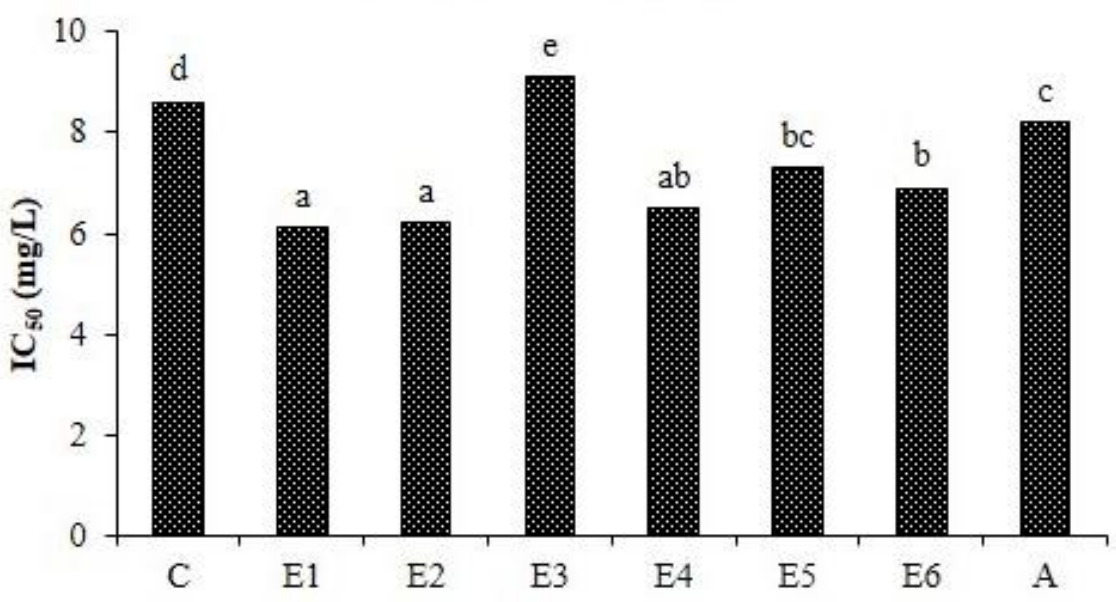

Figure 4. Anti-proliferation and anti-tyrosinase activity of cranberry pomace fractions

$\mathrm{IC}_{50}(\mathrm{mg} / \mathrm{L})$, concentration for inhibiting $50 \%$ of activity, $(\mathrm{P}<0.05), \mathrm{n}=3$. C, crude; E1-E6, ethanol based fractions; A1: acetone based fractions pooled together. Different letters indicate that values are statistically different $(\mathrm{p}<0.05)$.

Crude extract was found to be less effective in terms of its anti-proliferative activity, followed by fractions A, E5, and E6. This was however, different from what was observed in the case of anti-oxidant capacity data, where fractions E4 and E5 came out be the most effective in exhibiting anti-oxidant capacities. The relationship between the quantity of total phenolic contents and the anti-proliferative concentration was not established for all the tested fractions. For instance, it was observed that the total phenolic contents of the CP fractions containing anthocyanins or flavonols (E4-E5) were very high (Figure 2); however, the concentrations required against HepG2 cancer cells were significantly higher than that of other fractions with lower phenolic contents (E-E3). A plausible reason for this could be that the anti-proliferative activity of different fractions might depend on some main bioactive components and their interaction or synergistic effects. Our data were in agreement to results reported by $\mathrm{Vu}$ et al [2], 
which showed that the fractions containing the highest total phenolic content were not effective in inhibiting the growth of colon cancer cells.

\section{Anti-tyrosinase activity}

The tyrosinase activity of CP fractions was determined using the L-tyrosine oxidation assay. The absorbance at $490 \mathrm{~nm}$ decreases as a result of the reaction of melanin synthesis was interrupted. The results were expressed in $\mathrm{IC}_{50}$ value (Figure 4). All of the fractions showed strong antityrosinase activities and the activity of the given fractions increased with increasing concentrations, i.e. $10-500 \mathrm{mg} / \mathrm{L}$. The $\mathrm{IC}_{50}$ values of fractions were in the increasing order: $\mathrm{E} 1$ (6.1) < E2 (6.2) < E4 (6.5) < E6 (6.9) < E5 (7.3) < A (8.2) < C (8.6) < E3 (9.1) mg/L. Similar to anti-proliferation results, fractions E1 and E2 (rich in phenolic acids and catechins) were found to be the most effective in terms of inhibiting tyrosinase activity. This trend was followed by fraction E4 and E5 (enriched in flavonols). However, crude extract was found to be least effective in inhibiting tyrosinase activity.

In addition to the phenolic compounds determined in our study, cranberry is also known to be rich in arbutin, a potent antioxidant with a hydroquinone moiety that inhibits the activity of tyrosinase and inhibits melanosome maturation [25]. The low $\mathrm{IC}_{50}$ values of $\mathrm{CP}$ fractions are indicative of the presence of highly bioactive compounds against tyrosinase inhibition. The modulation of human skin pigmentation has been in demand for cosmetic and pharmaceutical applications for many years. Hydroquinones, kojic acid, and their derivatives are some of the whitening agents commonly used. However, these products have also been associated with many side effects. Our study is the first to present the anti-tyrosinase activity of CP based fractions in vitro. The $\mathrm{CP}$ being rich in tannins may employ the characteristic feature that tannins ability to bind with proteins as a mechanism of tyrosinase inhibitors. These interesting results would provide information regarding the feasibility of developing a natural $\mathrm{CP}$-based extract as a source of cosmetic ingredient with a skin-whitening and/or anti-aging property.

\section{CONCLUSIONS}

The results from this study showed that $\mathrm{CP}$ fractions contain a wide variety of bioactive compounds, including flavonols, flavan-3-ols, phenolic acids, anthocyanins, and proanthocyanidins. Fractions E4 and E5 (rich in flavonols and anthocyanins), followed by A1 (rich in proanthocyanidins) exhibited strong anti-oxidant capacity as determined by FRAP and DPPH assays. The CP fractions demonstrated a dose-dependent inhibition of human hepatocellular carcinoma HepG2 cells, with E1 (rich in phenolic acids and flavan-3-ols with $\mathrm{IC}_{50}$ $=7.8 \mathrm{mg} / \mathrm{L}$ ) presenting the highest inhibitory activity. Additionally, a similar concentration of E1 and $\mathrm{E} 2\left(\mathrm{IC}_{50}=6.1-6.2 \mathrm{mg} / \mathrm{L}\right)$, followed by $\mathrm{E} 4\left(\mathrm{IC}_{50}=6.9\right)$ demonstrated the potent antityrosinase inhibitory activity. Overall, all fractions of CP exerted biological activity greater than that demonstrated by the crude extract. To our knowledge, this is the first study to demonstrate the potential of CP fractions against human liver cancer cells and anti-tyrosinase activity in vitro. Nonetheless, further studies are required to confirm the mechanisms (apoptosis or necrotic) in which these fractions arrest the growth of human liver cancer cell lines. The anti-proliferative activity of CP fractions may be used with other anticancer agents as adjuvants to have synergistic 
or additive actions in treatment of liver cancer. These results suggest that $\mathrm{CP}$, instead of being land disposed, could be exploited as a potential source of natural antioxidant and other health promoting biologically active agents.

Abbreviation: CP, cranberry pomace; DMAC, dimethylcinnamaldehyde; DMSO, dimethyl sulfoxide; DPPH, 2,2-Diphenyl-1-picrylhydrazyl; EMEM, Eagle's Minimum Essential Medium; ESI, electrospray ionization; FRAP, ferric reducing antioxidant power; GAE, gallic acid equivalents; HepG2, human hepatocellular carcinoma; ME, malvidin-3-glucoside equivalents; MTS, 3-(4, 5-dimethylthiazol-2-yl)-5-(3-carboxymethoxyphenyl)-2-(4-sulfenyl)-2H tetrazolium; QE, quercetin equivalents; TE, Trolox equivalents; UPLC-MS, ultra-high performance liquid chromatography mass spectrometry.

Competing of interests: The authors hereby declare no conflict of interests.

Authors contributions: The principal investigator, HPVR, designed the experimental approach and contributed to the manuscript writing. Research associates, IP and SVN, conducted the experiments, analyzed the data and wrote the first draft of the manuscript.

Acknowledgments and funding: The authors acknowledge the financial support from the Natural Sciences and Engineering Council (NSERC) of Canada.

\section{REFERENCES}

1. Statistics Canada 2015: Table 001-0009 - Area, production and farm gate value of fresh and processed fruits, by province, annual, CANSIM (database). (Accessed on October $20^{\text {th }}$, 2015). Available at: http://www5.statcan.gc.ca/cansim/a26?lang=eng\&id=10009.

2. Vu KD, Carlettini H, Bouvet J, Côté J, Doyon G, Sylvain JF and Lacroix M: Effect of different cranberry extracts and juices during cranberry juice processing on the antiproliferative activity against two colon cancer cell lines. Food Chem 2012, 132:959967.

3. Roopchand DE, Krueger CG, Moskal K, Fridlender B, Lila MA, Raskin I: Foodcompatible method for the efficient extraction and stabilization of cranberry pomace polyphenols. Food Chem 2013, 141: 3664-9.

4. White BL, Howard LR, Prior RL: Polyphenolic composition and antioxidant capacity of extruded cranberry pomace. J Agric Food Chem 2010, 58:4037-42.

5. White BL, Howard LR, Prior RL: Proximate and polyphenolic characterization of cranberry pomace. J Agric Food Chem 2010, 58:4030-4036.

6. Neto CC: Cranberry and its phytochemicals: a review of in vitro anticancer studies. J. Nutr 2007, 137:186S-193S.

7. Caillet S, Lorenzo G, Côté J, Sylvain J and Lacroix M: Free radical-scavenging properties and antioxidant activity of fractions from cranberry products. Food Nutr Sci 2012, 3:337347.

8. Ou K, Percival SS, Zou T, Khoo C and Gu L: Transport of cranberry A-type procyanidin dimers, trimers, and tetramers across monolayers of human intestinal epithelial Caco-2 cells. J Agric Food Chem 2012, 60:1390-1396. 
9. Guthrie N, Effect of cranberry juice and products on human breast cancer cell growth. FASEB J 2000, 14:A771.

10. Singleton V and Rossi J: Colorimetry of total phenolics with phosphomolybdicphosphotungstic acid reagents. Am J Enol Vitic 1965, 16:144-158.

11. Rupasinghe HPV, Huber GM, Embree C and Forsline PL: Red-fleshed apple as a source of functional beverages. Can J Plant Sci 2010, 90:95-100.

12. Lee J, Durst RW and Wrolstad RE: Determination of total monomeric anthocyanin pigment content of fruit juices, beverages, natural colorants, and wines by the $\mathrm{pH}$ differential method: collaborative study. J AOAC Int 2005, 88:1269-1278.

13. Prior RL, Fan E, Ji H, Howell A, Nio C, Payne MJ and Reed J: Multi-laboratory validation of a standard method for quantifying proanthocyanidins in cranberry powders. J Sci Food Agric 2010, 90:1473-1478.

14. Bhullar KS and Rupasinghe HPV: Antioxidant and cytoprotective properties of partridgeberry polyphenols. Food Chem 2014, 168:595-605.

15. Rupasinghe HPV, Yu LJ, Bhullar KS, and Bors B: Haskap (Lonicera caerulea): A new berry crop with high antioxidant capacity. Can J Plant Sci 2012, 92:1311-1317.

16. Benzie IF and Strain JJ: The ferric reducing ability of plasma (FRAP) as a measure of "antioxidant power": The FRAP assay. Anal Biochem 1996, 239:70-76.

17. Lu Y and Foo LY: Antioxidant and radical scavenging activities of polyphenols from apple pomace. Food Chem 2000, 68:81-85.

18. Nair SVG, Ziaullah, and Rupasinghe HPV: Fatty acid esters of phloridzin induce apoptosis of human liver cancer cells through altered gene expression. PLOS ONE 2014, 9(9): e107149.

19. Sirat HM, Rezali MF and Ujang Z: Isolation and identification of radical scavenging and tyrosinase inhibition of polyphenols from Tibouchina semidecandra L. J Agric Food Chem 2010, 58:10404-10409.

20. Ziaullah, Bhullar KS, Warnakulasuriya SN, Rupasinghe, HPV: Biocatalytic synthesis, structural elucidation, antioxidant capacity and tyrosinase inhibition activity of long chain fatty acid acylated derivatives of phloridzin and isoquercitrin. Bioorg Med Chem 2013, 21:684-692.

21. Côté J, Caillet S, Doyon G, Sylvain JF and Lacroix M: Analyzing cranberry bioactive compounds. Crit Rev Food Sci Nutr 2010, 50:872-888.

22. Côté J, Caillet S, Doyon G, Sylvain JF and Lacroix M: Bioactive compounds in cranberries and their biological properties. Crit Rev Food Sci Nutr 2010, 50:666-679.

23. Zheng W and Wang SY: Oxygen radical absorbing capacity of phenolics in blueberries, cranberries, choke-berries, and lingonberries. J Agic Food Chem 2003, 51:502-509.

24. Puangpraphant S, Berhow MA, Vermillion K, Potts G and Mejia EG: Dicaffeoylquinic acids in Yerba mate (Ilex paraguariensis St. Hilaire) inhibit NF-kB nucleus translocation in macrophages and induce apoptosis by activating caspases- 8 and-3 in human colon cancer cells. Mol Nutr Food Res 2003, 55:1-14.

25. Sugimoto K, Nishimura T, Nomura K, Sugimoto K and Kuriki T: Inhibitory effects of alpha-arbutin on melanin synthesis in cultured human melanoma cells and a threedimensional human skin model. Biol Pharm Bull 2004, 27:510-514. 


\section{APPENDIX A}

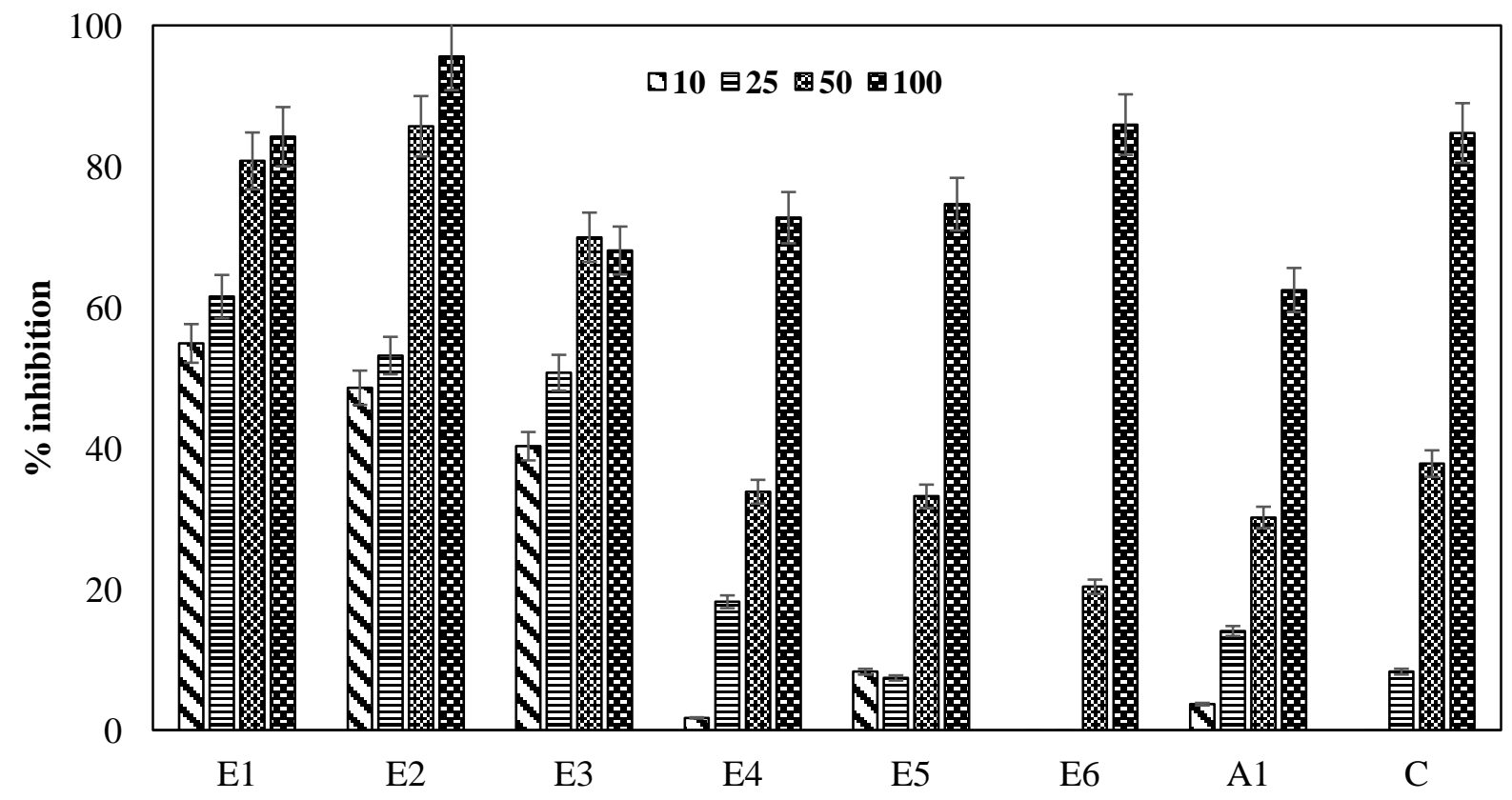

Figure 1S: Dose dependent relationship between different concentrations of cranberry pomace fractions $(10-100 \mathrm{mg} / \mathrm{L})$ and percentage inhibition of human hepatocellular carcinoma (HepG2) proliferation.

Bars represent standard errors $(n=3)$.

C, crude; E1-E6, ethanol based fractions; A1: acetone based fractions pooled together

\section{APPENDIX B}

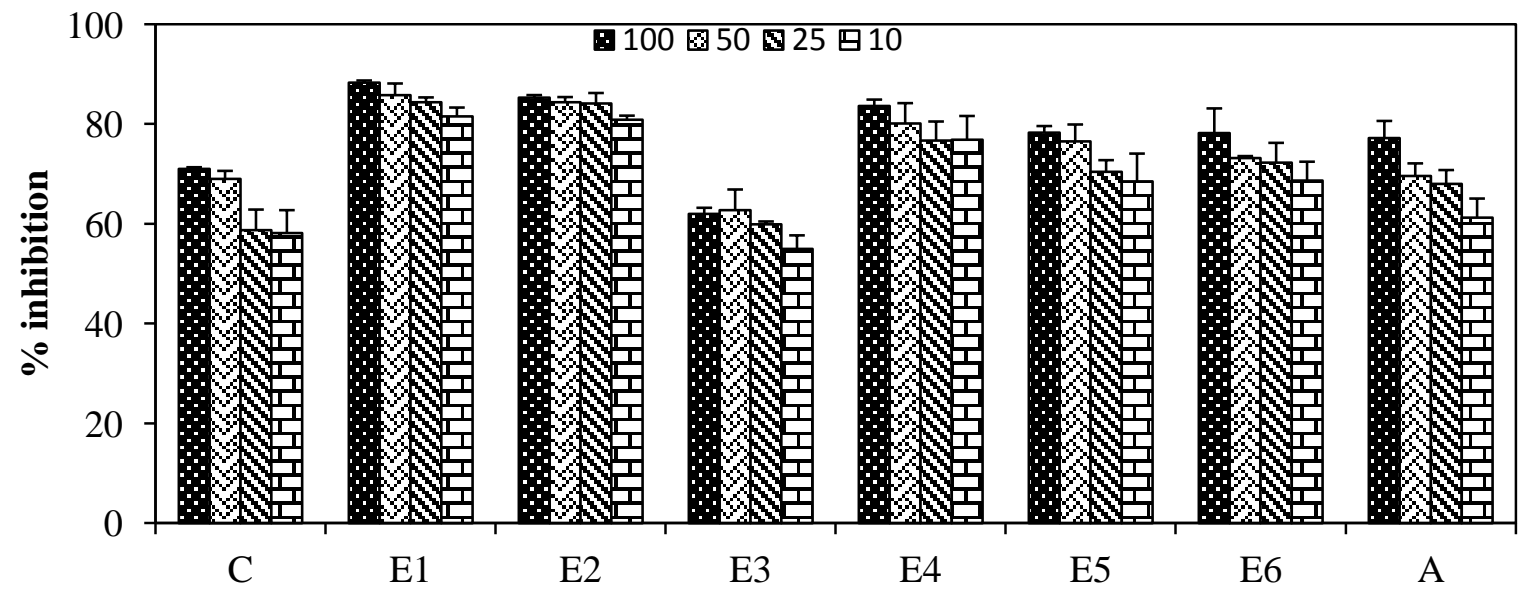

Figure 2S: Dose dependent relationship between different concentrations of cranberry pomace fractions $(10-100 \mathrm{mg} / \mathrm{L})$ and percentage inhibition of tyrosinase activity in vitro.

Bars represent standard errors $(n=3)$.

C, crude; E1-E6, ethanol based fractions; A1: acetone based fractions pooled together 